\author{
RESEARCH ARTICLE \\ 10.1029/2019JC015354 \\ Key Points: \\ - Ocean surface waves are attenuated \\ by sea ice, particularly at high \\ frequencies \\ - The preferential loss of \\ high-frequency energy causes a \\ narrowing of the scalar wave \\ spectrum in sea ice \\ - The narrow-banded waves have \\ more prominent group structures, \\ which can be explained by linear \\ mechanisms
}

Supporting Information:

- Supporting Information S1

Correspondence to:

J. Thomson,

jthomson@apl.washington.edu

Citation:

Thomson, J., Gemmrich, J., Rogers, W. E., Collins, C. O., \& Ardhuin, F. (2019). Wave groups observed in pancake sea ice. Journal of Geophysical Research: Oceans, 124, 7400-7411. https://doi.org/10.1029/ 2019JC015354

Received 8 JUN 2019

Accepted 23 SEP 2019

Accepted article online 16 OCT 2019

Published online 7 NOV 2019

\section{Wave Groups Observed in Pancake Sea Ice}

\author{
Jim Thomson ${ }^{1} \mathbb{D}$, Johannes Gemmrich ${ }^{2} \mathbb{D}$, W. Erick Rogers ${ }^{3}$, Clarence O. Collins ${ }^{4} \mathbb{D}$, \\ and Fabrice Ardhuin 5 (D) \\ ${ }^{1}$ Applied Physics Laboratory, University of Washington, Seattle, WA, USA, ${ }^{2}$ University of Victoria, Victoria, BC, \\ Canada, ${ }^{3}$ Naval Research Laboratory, Stennis Space Center, MS, USA, ${ }^{4}$ US Army Research and Development Center, \\ Coastal Hydraulics Laboratory, Duck, NC, USA, ${ }^{5}$ Univ. Brest, CNRS, Ifremer, IRD, Laboratoire d'Oceanographie \\ Physique et Spatiale, Brest, France
}

\begin{abstract}
Ocean surface waves propagating through sea ice are scattered and dissipated. The net attenuation occurs preferentially at the higher frequencies, and thus the spectral bandwidth of a given wave field is reduced, relative to open water. The reduction in bandwidth is associated with an increase in the groupiness of the wave field. Using Surface Wave Instrument Float with Tracking buoy data from the 2015 Arctic Sea State experiment, bandwidth is compared between pancake ice and open water conditions, and the linkage to group envelopes is explored. The enhancement of wave groups in ice is consistent with the simple linear mechanism of superposition of waves with narrowing spectral bandwidth. This is confirmed using synthetic data. Nonlinear mechanisms, which have been shown as significant in other ice types, are not found to be important in this data set.
\end{abstract}

\section{Introduction}

A universal feature of ocean surface waves is the propagation of waves in groups. In addition to sets of large waves posing a danger to ships and structures at sea, wave groups are important to several physical process at the ocean surface. In deep water, Banner and Pierson (2007) found that wave groups modulate breaking. This was later shown to be related to the slowing of crests and thus the local accumulation of wave energy in groups Banner et al. (2014). Gemmrich and Thomson (2017) found that rogue waves were tied closely to wave groups. van den Bremer and Taylor (2015) showed that wave groups are related to Lagrangian transport. Sullivan et al. (2018) found that wave groups can induce unsteadiness in the wind stress, because flow separation in the atmospheric boundary layer (and thus the form drag) is sensitive to the local wave slope.

In the purely linear mechanism, wave groups arise from the random superposition of neighboring frequency $f$ components in a wave field. This is related to the bandwidth of a given wave energy spectrum $E(f)$, which can be defined as (Longuet-Higgins, 1984)

$$
v=\left(\frac{m_{0} m_{2}}{m_{1}^{2}}-1\right)^{\frac{1}{2}}
$$

where $m_{n}$ are the $n$th moments of the scalar wave energy spectrum defined as $\int f^{n} E(f) d f$. There are several other definitions of bandwidth defined in the literature; the results that follow are incentive to the choice of definition. Phase information is important for the formation of wave groups. A spectrum with a wide bandwidth will have almost no group structure because the superposition of many wave components with random phase will be incoherent; thus, the destructive and constructive interference patterns do not form. A spectrum with a narrow bandwidth will have a strong group structure, because components will interact with phase coherence; two neighboring components will have a "beat" frequency equal to the difference in their primary frequencies, and there will be few other interactions to obscure the signal.

There are also nonlinear mechanisms for the emergence of wave groups, such as modulational instability (Benjamin \& Feir, 1967). This is a four-wave interaction process that can make a narrow-banded wave spectrum unstable. In sea ice, related theoretical four-wave interactions and instabilities have been identified, 
with associated changes to the deep-water wave dispersion relation (Liu \& Mollo-Christensen 1988; Polnikov \& Lavrenov 2007). A common metric used to assess the degree of nonlinearity is wave steepness,

$$
\epsilon=\sqrt{k_{p}^{2}\left\langle\eta^{2}\right\rangle}
$$

where $k_{p}$ is the wavenumber at the peak of the spectrum and $\left\langle\eta^{2}\right\rangle$ is the variance of the sea surface elevation $\eta$. The brackets $\langle$ Error〉 indicate an average over a short time span of raw wave measurements, typically 10 to $30 \mathrm{~min}$. The ratio of steepness to bandwidth is the Benjamin-Feir Index (BFI), Janssen (2003)

$$
B F I=\sqrt{2}\left(\frac{\epsilon}{v}\right)
$$

and modulational instability is expected for values of $B F I>1$. This threshold is somewhat dependent on the details in defining the BFI, but these do not change the overall interpretation of trends or relative changes in $B F I$ (Serio et al., 2005). This instability is derived for narrow directional seas, though some have defined an effective BFI for directional seas (Waseda et al., 2009), which would slightly reduce the values reported here. The BFI is related to the kurtosis (Janssen, 2003)

$$
\kappa=\frac{\left\langle\eta^{4}\right\rangle}{\left\langle\eta^{2}\right\rangle^{2}},
$$

because both include measures of nonlinearity. Specifically, the kurtosis uses the second moment of the surface elevations to normalize the fourth moment of the surface elevations $\eta$.

Nonlinearity also can cause wave groups via three-wave interactions; however, the theoretical basis is limited to shallow water. Previous studies (Elgar et al., 1984) have found nonlinearity to be significant in the formation of wave groups in shallow water, though in deeper water, the same study concluded that linear superposition of components with random phase was sufficient to reproduce most of the groups observed. Such nonlinearities are often diagnosed with the weighted skewness

$$
\gamma=\frac{\left\langle\eta^{3}\right\rangle}{\left\langle\eta^{2}\right\rangle^{\frac{3}{2}}},
$$

which is the normalized third moment of the surface elevations $\eta$. Again, the brackets \langle\rangle are an average over 10 to $30 \mathrm{~min}$, depending on the measurement platform.

Wave groupiness in sea ice has been previously observed (Collins et al., 2015), including application of the BFI metric to suggest that the nonlinear process of modulational instability in the presence of high compression stresses is responsible for the wave groups (Liu \& Mollo-Christensen, 1988). However, the theoretical framework for this process is restricted to wave spectra with narrow bandwidth and narrow directional spread, and we expect such instabilities to be reduced in the broadbanded and directionally rich waves observed in the natural ocean (Toffoli et al., 2010). Furthermore, Collins et al. (2015) note that accurate determination of wave steepness (and thus BFI) from buoy data requires knowledge of the dispersion relationship in sea ice, which was not measured in Collins et al. (2015). More broadly, we note that nearly all wave-ice measurements and models indicate preferential attenuation at high frequencies that would be sufficient to narrow the spectrum and hence, increase groupiness. Collins et al. (2015) note that groupiness may be particularly important for ice breakup and evolution of the ice floe size distribution, as groups of large waves are more likely to exceed flexure criteria.

Here, we apply a new dataset to evaluate linear and nonlinear mechanisms for the emergence of wave groups in sea ice. The new dataset spans a range of pancake ice and open water conditions, with associated changes in observed spectral bandwidth plus an explicit confirmation of the deep water dispersion relation throughout the dataset (Collins et al., 2018). We evaluate the linear mechanisms using synthetic data representing linear waves with a known bandwidth, and we evaluate the nonlinear mechanisms using the aforementioned metrics of wave steepness, skewness, and kurtosis.

\section{Methods}

\subsection{Wave Observations}

Wave data were collected from 11 to 14 October 2015 in the central Beaufort sea as part of the Arctic Sea State program (Thomson et al., 2018). Figure 1 shows the locations of freely drifting Surface Wave Instrument 


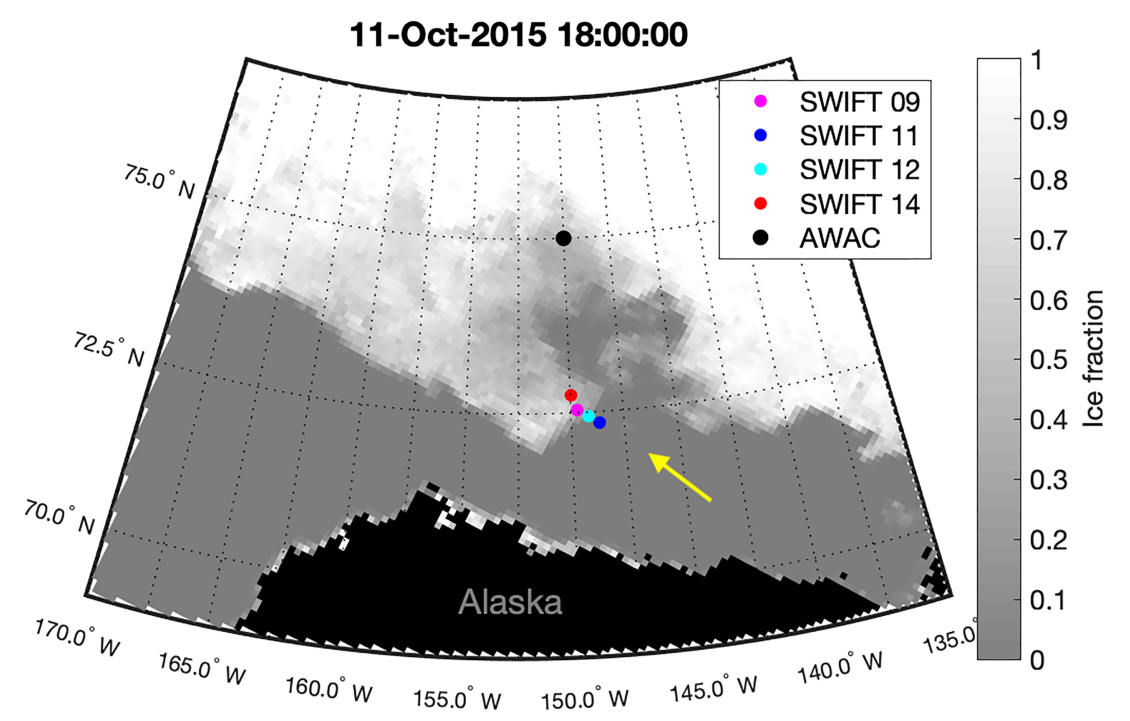

Figure 1. Location of SWIFT buoy and Acoustic Wave And Current mooring measurements, along with the coast of Alaska and sea ice concentration on 11 October 2018 at 18:00 UTC. The yellow arrow shows the direction of wave propagation.

Float with Tracking (SWIFT) buoys and a moored subsurface Acoustic Wave And Current (AWAC) sensor. The SWIFTs spanned a region from open water to nearly complete ice cover in the vicinity of $72^{\circ} \mathrm{N}, 150^{\circ}$ W. The AWAC mooring location was in complete ice cover, approximately $250 \mathrm{~km}$ from the ice edge at $75^{\circ}$ $\mathrm{N}, 150^{\circ} \mathrm{W}$ (Beaufort Gyre Observing System [BGOS] mooring A).

The SWIFTs measure waves using a combination of GPS velocities and vertical accelerations to produce the standard bulk and spectral wave parameters (Herbers et al., 2012; Thomson, 2012). Data are collected at $25 \mathrm{~Hz}$ in 512-s bursts every $12 \mathrm{~min}$. The first portion of the burst is screened because the data quality is poor until sufficient satellite signals are acquired. The raw vertical accelerations are rotated from the buoy reference frame to an earth reference frame (i.e., true vertical), and wave elevation time series $\eta(t)$ are estimated by double integrating the acceleration data. A high-pass filter is applied to avoid amplification of low frequency $(f<0.05 \mathrm{~Hz})$ noise in the double integration (Thomson et al., 2018).

The AWAC measures waves using a combination of a subsurface pressure measurement and an uplooking sonar to produce the standard bulk and spectral wave parameters (Pederson et al., 2007). Data are collected at $0.5 \mathrm{~Hz}$ in 1024-s bursts every $120 \mathrm{~min}$. The raw sonar ranges from the surface are the wave elevation time series $\eta(t)$.

Bulk wave parameters and wave frequency spectra are calculated for each burst of data (SWIFT and AWAC) following the details in Thomson et al. (2018), including estimation for directional spread (Kuik et al., 1988). The resulting spectra have 24 degrees of freedom and a frequency $f$ resolution of $0.0117 \mathrm{~Hz}$. The wavenumber at the peak of each spectrum, $k_{p}$, is determined from deep water dispersion, $(2 \pi f)^{2}=g k$. This choice is justified by the study of Collins et al. (2018), who show no significant deviations from deep water dispersion relation at the spectral peak across all of the observed ice conditions in this dataset.

\subsection{Wave Group Metrics}

A groupiness factor is defined for each 8-minute record as

$$
G F=\frac{\sigma_{S I W E H}}{\langle S I W E H\rangle},
$$

where the Smoothed Instantaneous Wave Energy History is defined as (Funke \& Mansard, 1980)

$$
S I W E H=\frac{1}{T_{p}} \int_{T_{p} / 2}^{-T_{p} / 2} \eta^{2}(t+\tau) Q(\tau) d \tau
$$



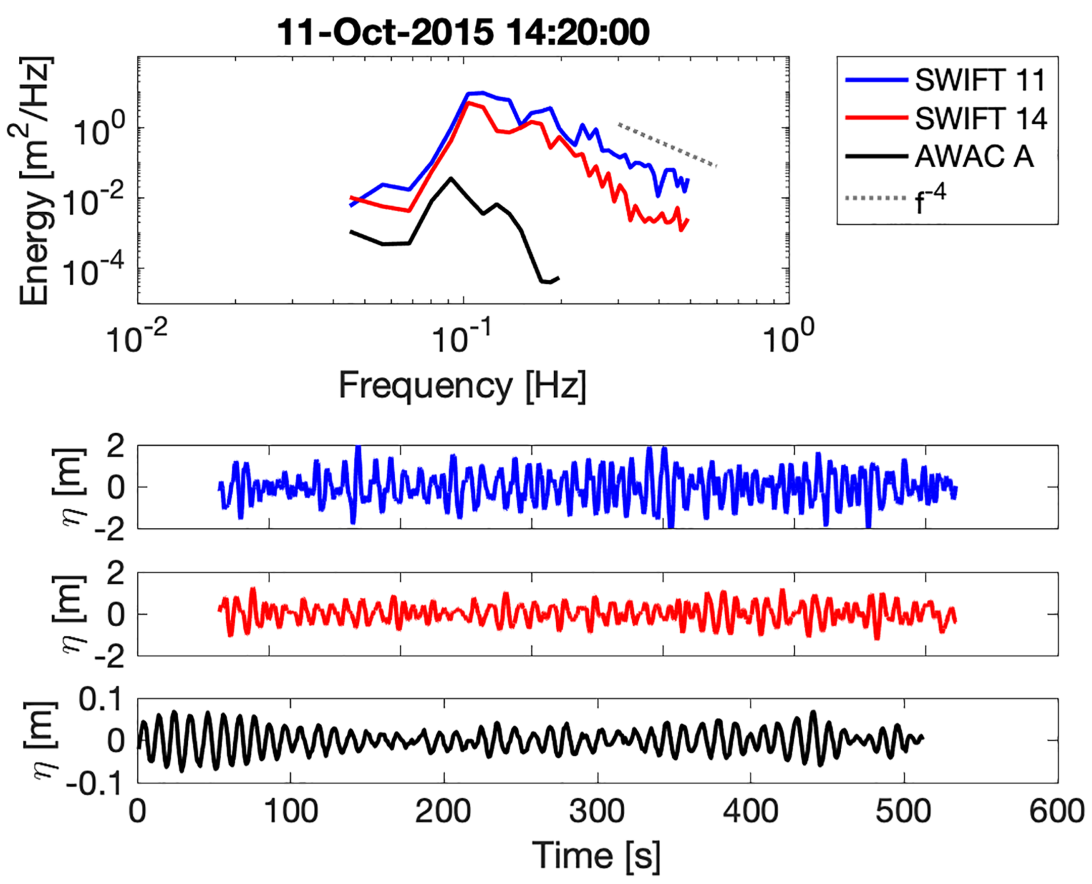

Figure 2. Example spectra and associated wave elevation time series from SWIFT buoys and Acoustic Wave And Current (AWAC) mooring on 11 October 2015 at 14:20 UTC. SWIFT 11 is in open water, SWIFT 14 is in variable ice cover, and the AWAC at mooring A is in complete ice cover. A dashed line indicates the spectral equilibrium range with frequency dependence $f^{-4}$.

and the brackets indicate an 8-min burst-average. The function $Q(\tau)$ is a Bartlett window with a length set by the peak wave period $T_{p}$, such that

$$
Q(\tau)=1-\frac{\tau}{T_{p}} \quad \text { for } \quad|\tau|<T_{p}
$$

and $Q(\tau)=0$ otherwise. This effectively traces the envelope of wave energy in a time series, and then $G F$ quantifies how much that envelope varies.

Figure 2 shows example scalar wave spectra and raw time series of sea surface elevations from the AWAC and from two SWIFT buoys. Spectra from SWIFT 11 in open water exhibit classic equilibrium frequency $f^{-4}$ dependence (Phillips, 1985) of the high frequency face, and the wave elevation time series do not have strong

\begin{tabular}{ll}
$\begin{array}{l}\text { Table 1 } \\
\text { Ice codes from }\end{array}$ & SWIFT buoy onboard images \\
\hline Ice Code & \\
\hline 1 & Open water \\
2 & Open water and trace pancakes \\
3 & Open water and frazil \\
4 & Open water and frazil with trace pancakes \\
5 & Pancakes and frazil, with open water breaks \\
6 & Pancakes and dark frazil, less open water breaks \\
7 & Pancakes and dark frazil, increasing concentration \\
8 & Pancakes and frazil, increasing concentration \\
9 & Pancakes with raised edges (20\%) and frazil, increasing concentration \\
10 & Pancakes with raised edges (> 40\%) and frazil, increasing concentration \\
11 & Pancakes with raised edges ( $>40 \%)$ and lighter frazil, increasing concentration \\
12 & Pancakes with raised edges and thicker frazil, highest concentration \\
\hline
\end{tabular}




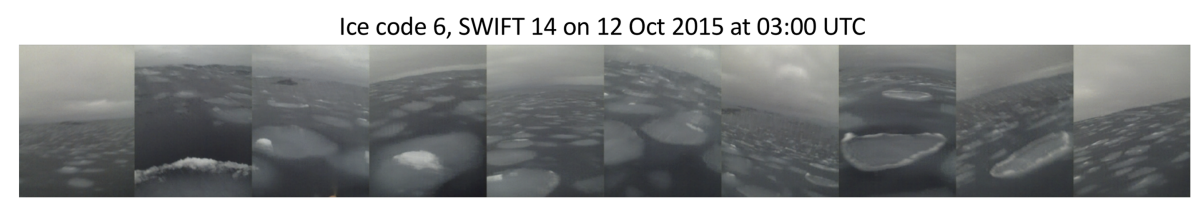

Ice code 7, SWIFT 14 on 12 Oct 2015 at 02:30 UTC

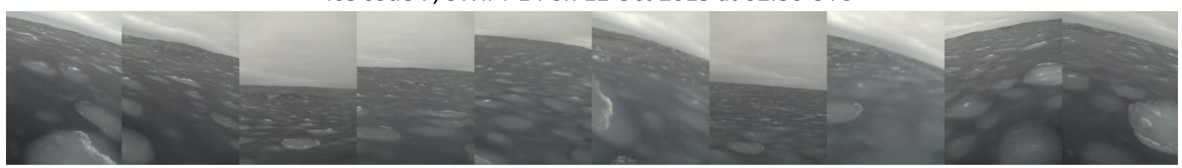

Ice code 8, SWIFT 14 on 11 Oct 2015 at 22:30 UTC

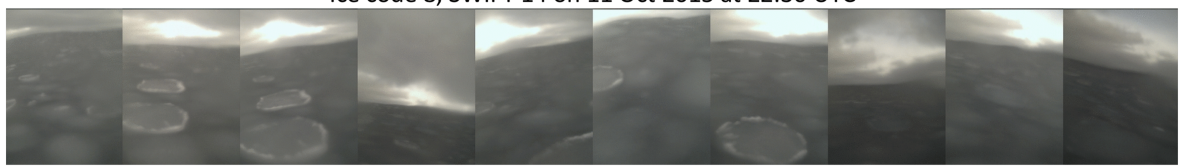

Ice code 9 , SWIFT 14 on 12 Oct 2015 at 00:30 UTC

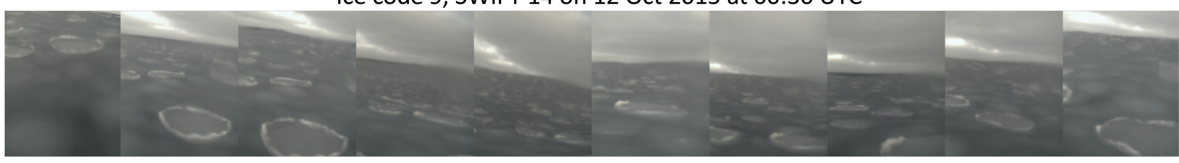

Ice code 12, SWIFT 14 on 12 Oct 2015 at 23:30 UTC

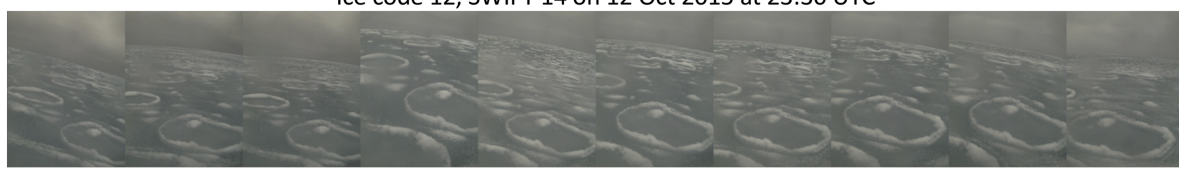

Figure 3. Example mosaic image compilations (10 images each) and subjective ice categories collected on SWIFT 14.

group structure. Spectra from SWIFT 14, located in partial ice cover, have reduced energy at high frequencies, and the wave elevation time series have modest group structure. Spectra from the AWAC, located in complete ice cover, have strong reductions at all frequencies (though still most significant at high frequencies), and the wave elevation time series has obvious wave group structure. The decreases in high-frequency energy for the AWAC and SWIFT 14, relative to SWIFT 11, are described by bandwidth values of $v=0.14$ and 0.28 , relative to $v=0.42$. The corresponding raw time series of surface elevation have groupiness factor values of $G F=0.8$ and 0.62 , relative to to $G F=0.58$. So clearly, there is a correlation, or correspondence, of ice cover to wave group structure. The question remains, is the simple linear explanation, that is, the emergence of coherent interference due to the narrowing of spectra, sufficient? Or is this a nonlinear process, that is, an instability initiated by the ice effect on wave dynamics?

\subsection{Ice Observations}

Ice observations are from images collected from the buoys, using a camera on a small mast (0.9-m elevation above the wave surface). These images are recorded every $4 \mathrm{~s}$ and then manually categorized in post-processing images compiled into mosaics. Table 1 presents the numerical codes used in manual categorization, and Figure 3 shows examples for the various pancake ice codes. Although subjective, these codes successfully differentiate between open water, frazil, and pancake ice. The images are only useful during daylight hours and only during the first half of the buoy deployments. In the second half of the deployments, most cameras became encased in ice as a result of freezing spray, and the images are too blurry to determine an ice code. These are the same ice codes used in Rogers et al. (2018).

Additional visual ice observations and ice thickness measurements were presented in Smith et al. (2018) and Smith and Thomson (2019). These are briefly summarized as follows: pancake ice floes of approximately 0.3 to $0.5-\mathrm{m}$ thickness, and 0.6 to $1-\mathrm{m}$ diameter formed rapidly during the event, with the highest concentration to the northwest portion of the buoy array. These pancakes melted during the later portion of the wave event, as result of heat released from the ocean (Smith et al., 2018). SWIFTs 11 and 12 were deployed in open water and frazil, then recovered from mostly open water with remnant pancake ice. SWIFTs 09 and 14 were deployed in high concentrations of pancake ice, then recovered in partially open water (14) and completely open water (09) with remnant pancake ice. No visual ice observations were collected for the AWAC at mooring A; however, Sentinel-1 satellite observations suggest complete ice cover throughout the event 

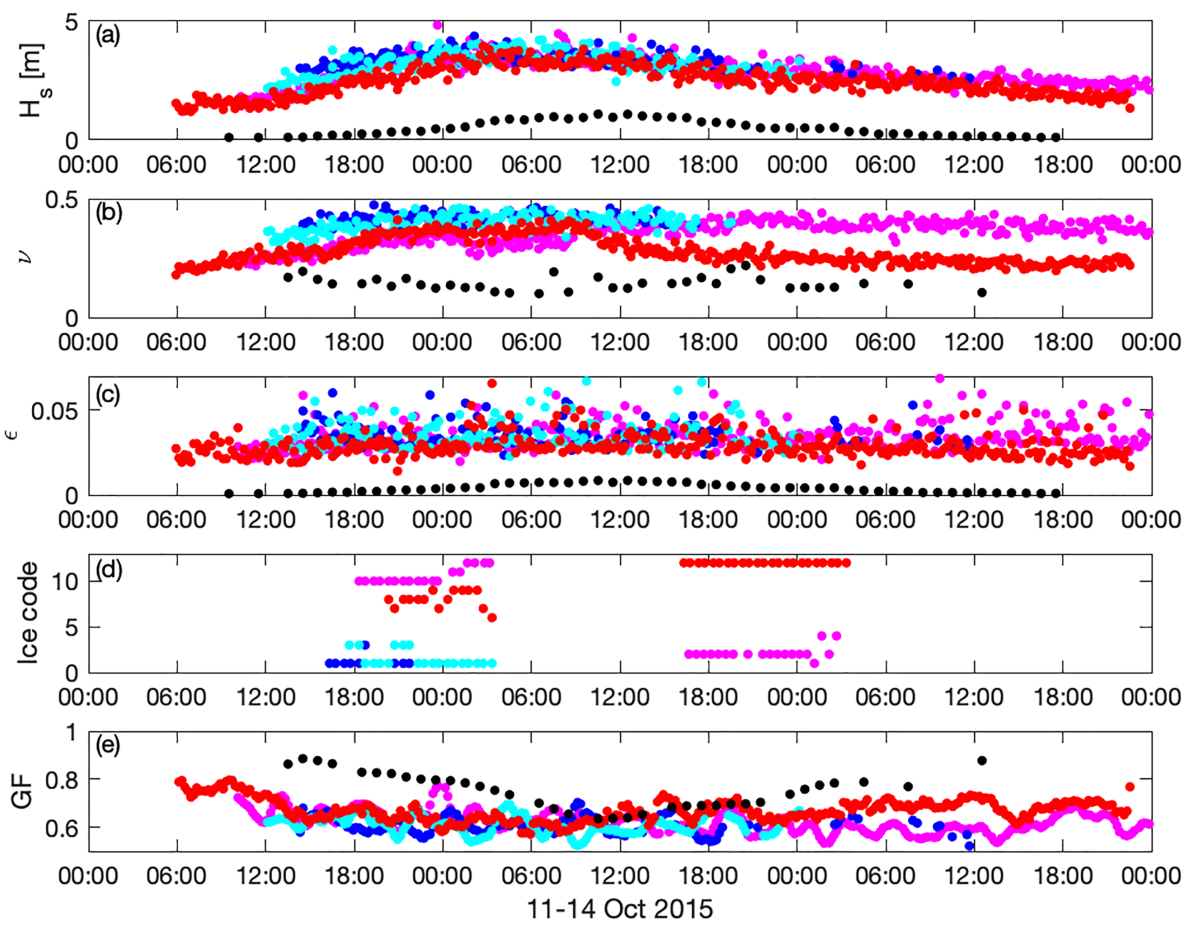

Figure 4. Time series of (a) significant wave height $H_{s}$, (b) spectral bandwidth $v$, (c) wave steepness $\epsilon$, (d) ice codes from the SWIFT images, and (e) groupiness factor GF. Colors indicate the observing platform, following the convention in Figure 1.

(Stopa et al., 2018). Comparison of the acoustic altimeter measurements and the pressure data, following the methods of Magnell et al. (2010), suggest ice thickness at the AWAC varied from 0.5 to $1 \mathrm{~m}$ during this event.

\section{Results}

Wave conditions during the event have been previously described in Rogers et al. (2016) and are only reviewed here. Strong winds from the southeast created an energetic wind sea, with a significant wave height around $H_{s}=4 \mathrm{~m}$ and a peak period around $T_{p}=10 \mathrm{~s}$. The directional spread at the peak period was around $35 \pm 10$ degrees throughout the event and independent of ice conditions. The event lasted for 3 days, and during this time, the ice conditions changed continually in the region of the SWIFTs. At first, strong heat loss from the ocean surface to the atmosphere caused ice formation (Persson et al., 2018). Then, ocean heat stored at depth was mixed to the surface, and much of the ice melted (Smith et al., 2018). Figure 1 uses the AMSR2 satellite ice product to show the ice conditions at the observation locations during the peak of the event, and Figure 4 show time series of the wave and ice conditions for each burst of raw data and each platform during the event.

Rogers et al. (2016) show preferential attenuation of high-frequency wave energy in sea ice during this event. This is further confirmed and expanded by Cheng et al. (2017), who calibrate a viscoelastic model to reproduce the observations. In the time series, the wave attenuation at the AWAC is severe, relative to the SWIFTs, because the location is much farther within the ice. The AWAC significant wave heights, bandwidth, and wave steepness are reduced, while the groupiness is increased, relative to the SWIFTs. There are also important variations within the buoy array. The bandwidth and wave steepness are always less for SWIFT 14 than the other SWIFTs, and the ice concentration around SWIFT 14 is always higher. Consistent with this, the groupiness is always increased at SWIFT 14 relative to the other SWIFTs. There is also significant variability of wave and ice conditions observed by a single buoy; SWIFT09 captures an evolution from high ice concentration (with low bandwidth and high groupiness) to low ice concentration (with high bandwidth and low groupiness).

These results are combined in Figure 5, which shows how spectral bandwidth $v$ and groupiness factor GF vary as a function of the Ice Codes derived from SWIFT buoy images. As suggested in the time series, the 

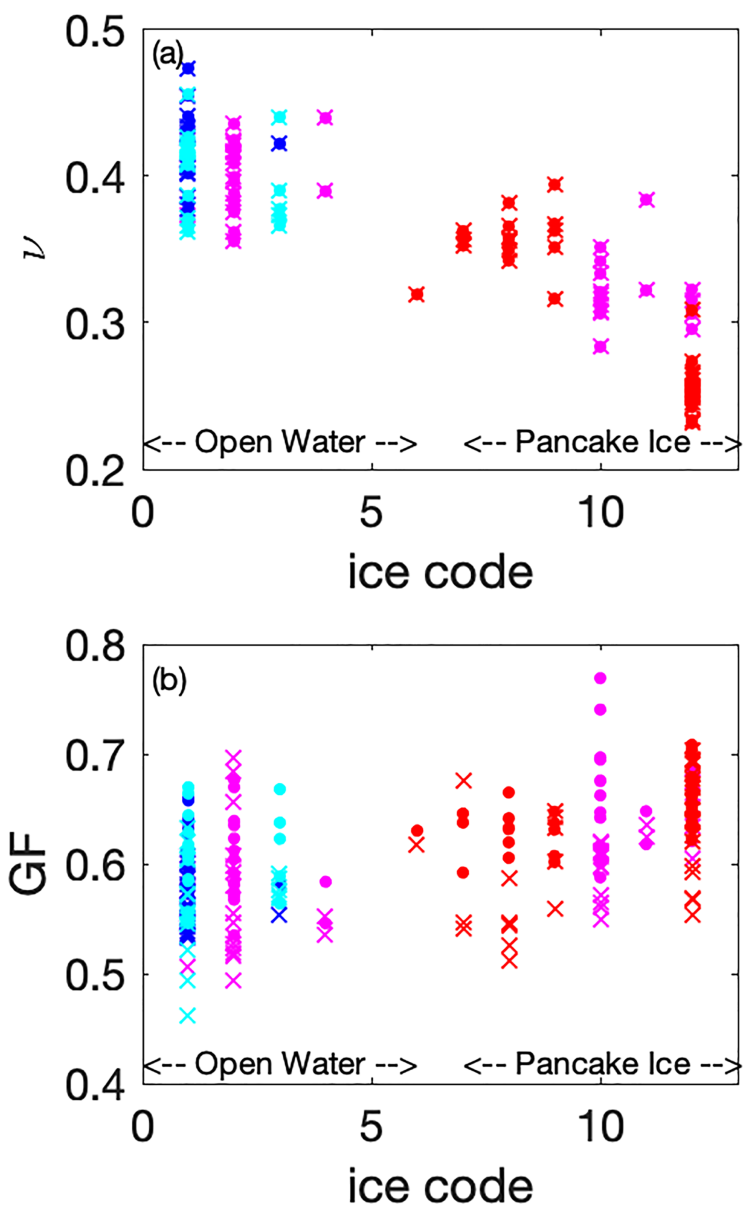

Figure 5. (a) spectral bandwidth $v$ and (b) groupiness factor $G F$ versus Ice Codes throughout the entire time series. Colors indicate the observing platform, following the convention in Figure 1. Small circles are from individual bursts of raw wave elevation data. Small crosses are from individual bursts of raw wave elevations reconstructed as a random sea with the same observed spectrum.

bandwidth values are reduced in the pancake ice, and the groupiness is increased in the pancake ice, relative to open water. In addition to results from observed wave elevation time series, Figure 5 includes results from synthetic wave data generated using the spec2sdat.m routine from the WAFO-group (2000) toolbox with observed spectra (i.e., random linear wave fields with the same bandwidth as the observations). The synthetic (linear) results are largely consistent with the observations, although the groupiness is slightly reduced compared with the observations. Elgar et al. (1985) found that group statistics were not sensitive to the choice of random sea reconstruction methods, and thus, this conclusion is unlikely to be a consequence of the particular WAFO implementation for generating the synthetic data. The essential property of the method is the absence of terms accounting for nonlinear interaction - this is purely a linear simulation.

The example raw time series and spectra shown in Figure 2 suggest that there is a strong correlation between groupiness factor $G F$ and bandwidth $v$, and Figure 5 suggests that this is dependent on ice type. This result is generalized in Figure 6 using the bandwidth $v$ (equation (1)) of all observed spectra and the groupiness factor $G F$ (equation (6)) of all observed wave elevation time series. Results are shown for the observations, as well as for the synthetic data corresponding to linear wave fields synthesized from the observed spectra. Observations and the synthetic data both show a strong trend of increased groupiness with decreasing bandwidth. The results from SWIFT 11 (in mostly open water throughout the event) and the AWAC (in complete ice cover throughout the event) are clustered at either end of the trend. The results from the other SWIFTs fill out the trend-those ice conditions varied throughout the event and produced a range of wave conditions. The synthetic results are largely consistent, and thus, linear mechanisms sufficiently reproduce the observed wave groups. 


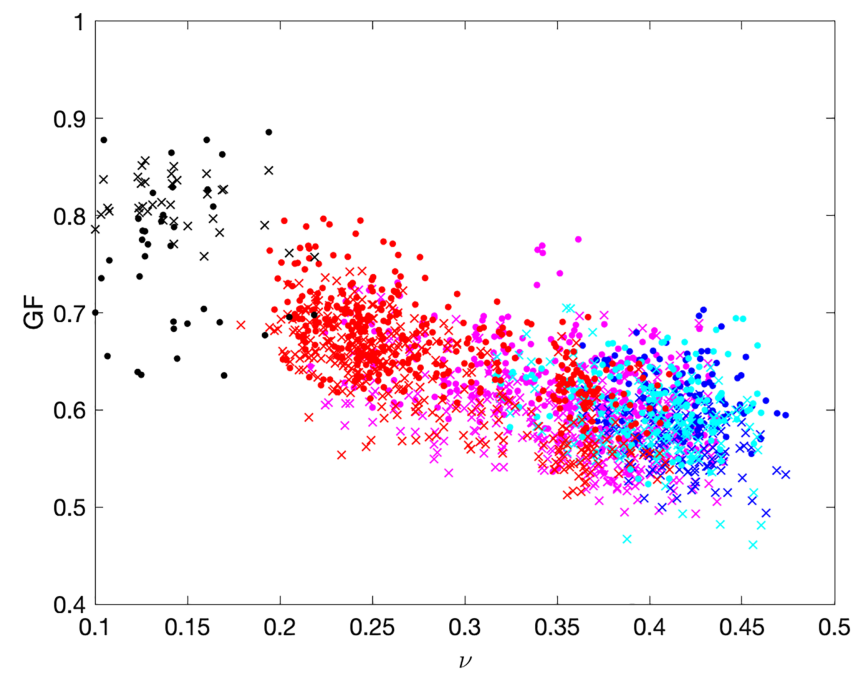

Figure 6. Groupiness factor $G F$ versus spectral bandwidth $v$ throughout the entire time series. Colors indicate the observing platform, following the convention in Figure 1. Small circles are from individual bursts of raw wave elevation data. Small crosses are from individual bursts of raw wave elevations reconstructed as a random sea with the same observed spectrum.

The possibility for groupiness associated with nonlinearity is assessed using the steepness of the observed waves in Figure 7. The steepness is much higher for the SWIFT buoys than for the AWAC observations, which may be partially explained by the limitations in the sampling frequency of the AWAC $\left(f_{s}=0.5 \mathrm{~Hz}\right.$, compared with $f_{s}=25 \mathrm{~Hz}$ for the SWIFT buoys). However, extrapolation of the observed wave spectra beyond the Nyquist frequency of $0.25 \mathrm{~Hz}$ does not change the steepness values significantly, suggesting that the low steepness values in the AWAC observations are mostly the result of wave attenuation through the ice. Within the SWIFT buoy observations, the steepness values have significant scatter and suggest only a weak negative correlation with observed groupiness. Across all platforms, the overall trend is for reduced groupiness with increasing wave steepness. This trend, though weak, is contrary to what one would expect if nonlinear mechanisms were responsible for the formation of wave groups (i.e., groupiness would increase with increasing wave steepness). The likely explanation is simple: wave steepness scales with wave energy, and these waves have less energy in sea ice. The more important mechanism appears to the be reduction

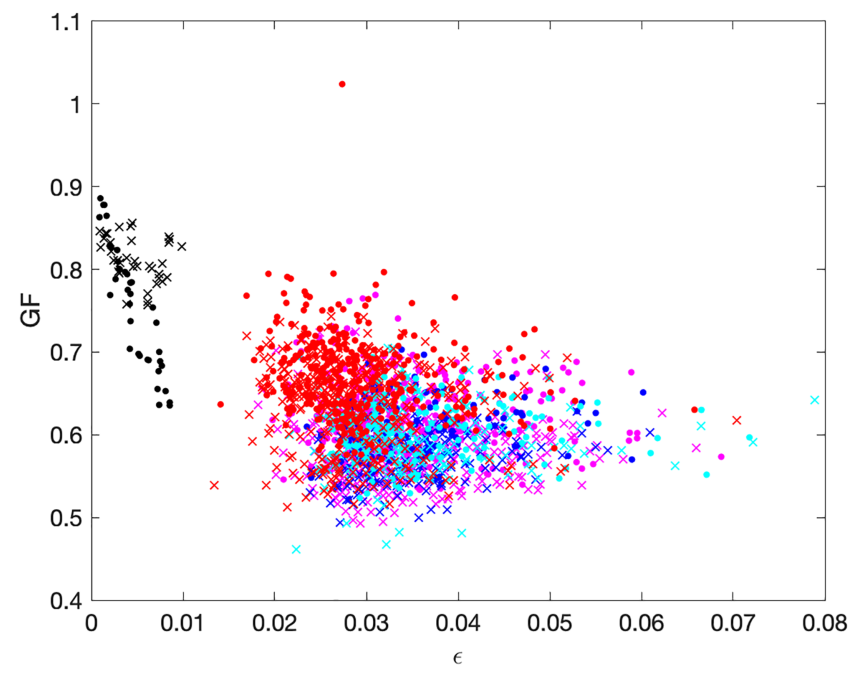

Figure 7. Groupiness factor $G F$ versus wave steepness $\epsilon$ throughout the entire time series. Colors indicate the observing platform, following the convention in Figure 1. Small circles are from individual bursts of raw wave elevation data. Small crosses are from individual bursts of raw wave elevations reconstructed as a random sea with the same observed spectrum. 


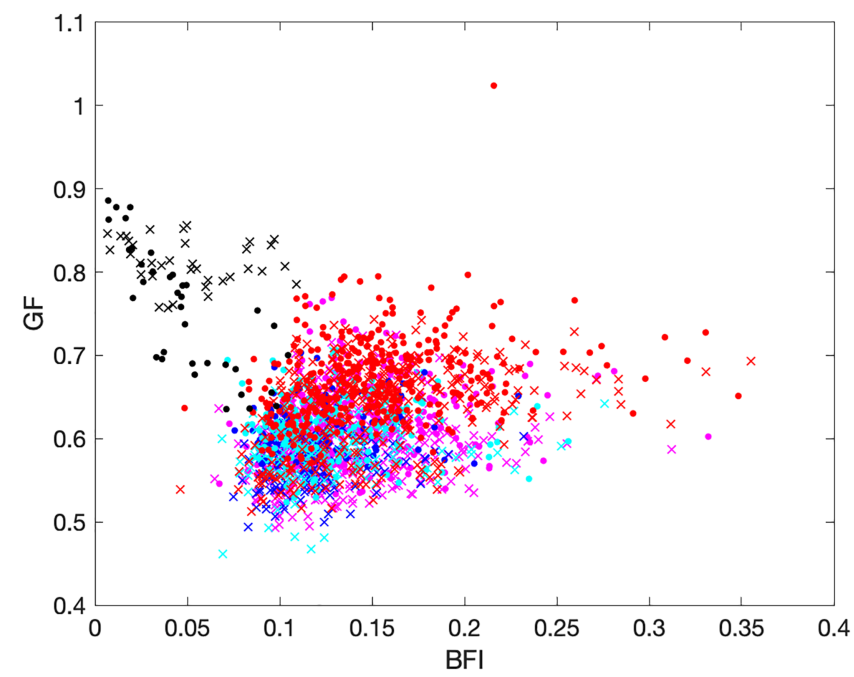

Figure 8. Groupiness factor $G F$ versus Benjamin-Feir Index $B F I$ throughout the entire time series. Colors indicate the observing platform, following the convention in Figure 1. Small circles are from individual bursts of raw wave elevation data. Small crosses are from individual bursts of raw wave elevations reconstructed as a random sea with the same observed spectrum.

of bandwidth in sea ice, in which groupiness emerges from simple linear superposition of coherent wave phases.

For completeness, the possibility for groupiness associated with nonlinearity is further assessed using the Benjamin-Feir Index in Figure 8. There is a notable difference between the BFI values from the AWAC observations and SWIFT buoys, which may again be partially a result of limitations in the sampling frequency of the AWAC. The overall trend is for reduced groupiness with increasing BFI, which, again, does not support the importance of nonlinear mechanisms. There is, however, a weak trend within the SWIFT observation for increased groupiness with BFI, but this is likely just a consequence of the linear dependence on bandwidth reduction (which is the denominator of the $B F I$ in equation (3)). Finally, we note that these values are all much lower than the instability criterion of $B F I>1$. The low $B F I$ values likely are related to the large directional spreads $\left(>35^{\circ}\right)$ of the observed directional wave spectra.

\section{Discussion}

We have found no support for nonlinear wave groups, which is not surprising given that the incident wave field was not very steep $(\epsilon \sim 0.05)$ in open water. For completeness, there are a few other nonlinear metrics that might affect the groupiness of the observed waves. These are drawn from the distributions of the raw sea surface elevation time series $\eta$. Example distributions are shown in Figure 9, using the same raw data
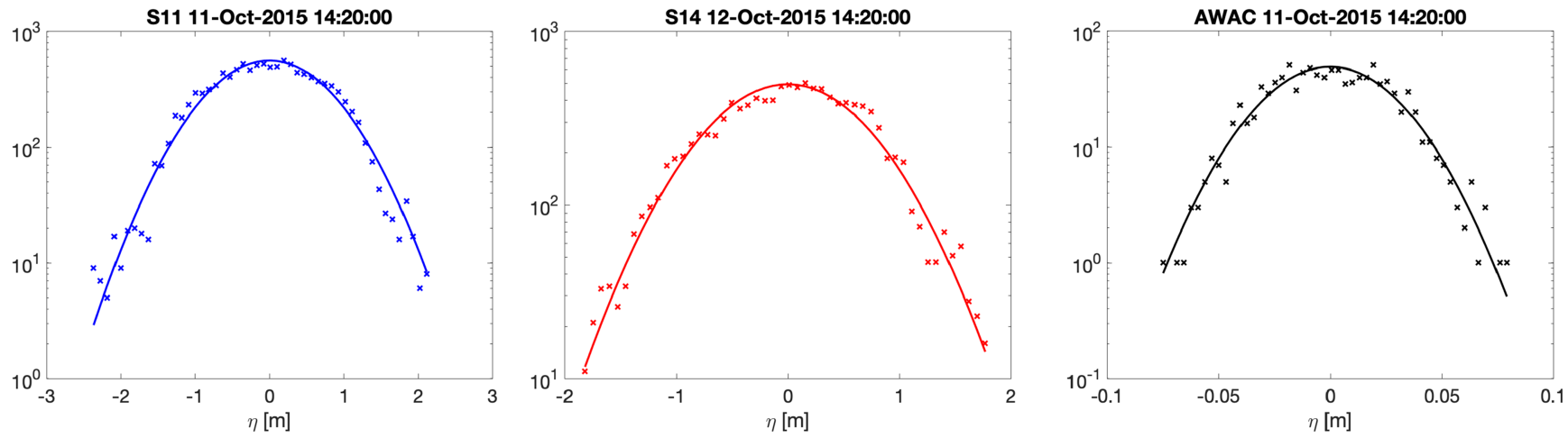

Figure 9. Example histograms and fitted Gaussian distributions of sea surface elevations for SWIFT 11, SWIFT 14, and the AWAC. This example is for the same raw data as the example spectra in Figure 2. 

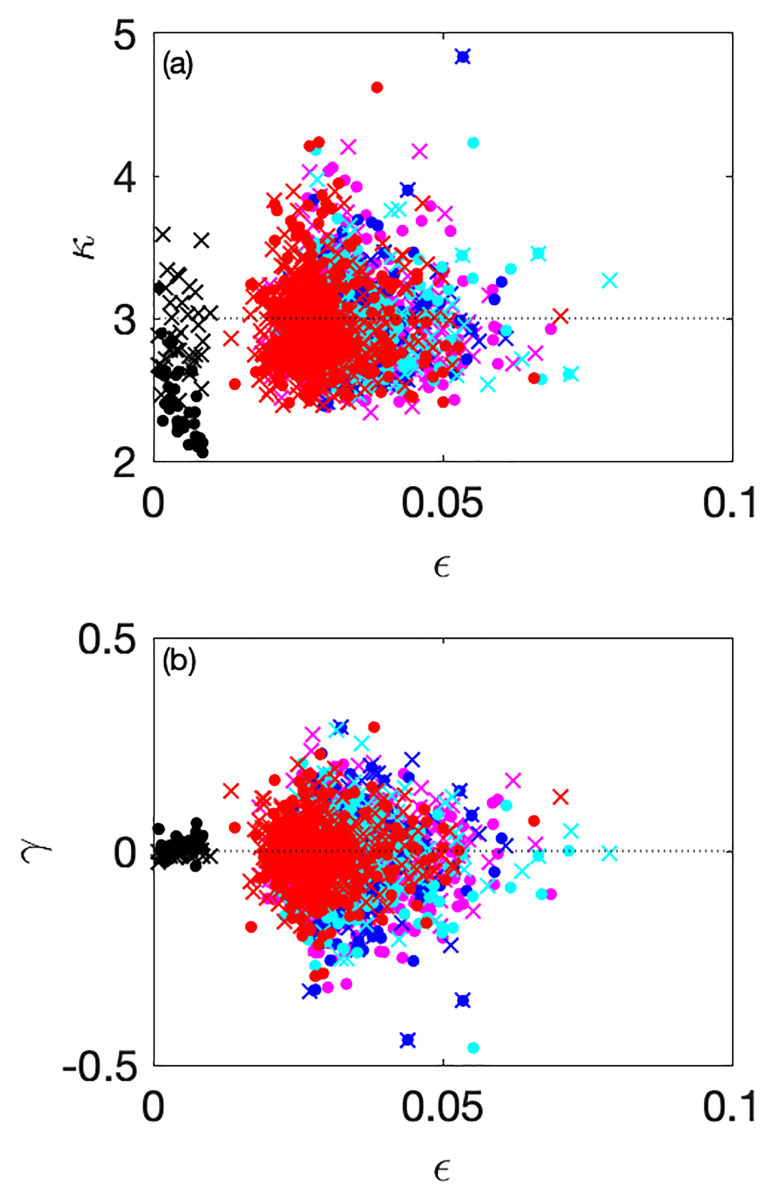

Figure 10. Wave asymmetry and kurtosis versus wave steepness throughout the entire event. Colors indicate the observing platform, following the convention in Figure 1. Small circles are from individual bursts of raw wave elevation data. Small crosses are from individual bursts of raw wave elevations reconstructed as a random sea with the same observed spectrum.

in Figure 2. Examples from the other bursts of raw data are available as supplemental material. At all three locations, surface elevation closely follows a Gaussian distribution, indicating a linear wave field.

The additional nonlinear metrics of wave skewness and kurtosis are shown in Figure 10. These are reduced for the AWAC measurements (again probably because of the sampling frequency limitation) and similar across the SWIFT observations. The skewness values are centered around 0 , and the kurtosis values are centered around 3. Thus, both values are as expected for a Gaussian sea surface. Such statistical measures of nonlinearity typically are calculated from much longer raw times series than the 12-min bursts of data used herein. The scatter in Figure 10 is thus not surprising, given the short records used, but it is retained to be consistent with the SWIFT processing and other published SWIFT data.

These higher order statistics and related mechanisms are important for wave probabilities. Although both linear and nonlinear mechanisms result in similar group structure, maximum wave height is expected to be different. This distinction may be important in determining wave effects on ice. As a wave propagates through ice, it induces a stress felt by the ice and the ice strains (bends and deforms) to support the wave form. Once this stress exceeds the flexural strength of the ice, the ice fractures and eventually breaks apart. The maximum stress expected is directly related to the maximum expected wave height that, in turn, is a function of the wave height probability distribution (PDF). If modulation instability was responsible for the group structure, one would also expect raised tail of the PDF, that is, an increased occurrence of larger than average waves and a larger overall maximum expected wave. This would be evident in the shapes of the PDFs and also in the higher order statistics. 
In general, wave ice interaction can be thought of in two primary regimes: scattering dominant-where ice floes are much larger than the characteristic wavelength of incident waves-and dissipation dominant, where the ice floes are smaller than the characteristic wavelength of incident waves. The data herein are certainly a dissipation dominant regime. Aside from this scaling argument, the material properties of sea ice are highly variable (Timco \& Weeks, 2010), and just as variable is the specific nature of the ice effect ice on wave propagation. Although a significant range of ice conditions were encountered during the Arctic Sea State project, our results are limited by considering the full scope of sea ice variability. While we did not observe anything which suggested nonlinear wave dynamics, it is not possible to rule them out in general.

Other studies (Collins et al., 2015; Liu \& Mollo-Christensen, 1988) have suggested strong nonlinear wave group effects in sea ice, in contrast to the results of the present study. We briefly compare the conditions under which wave-ice interaction occurred for Sea State and for Collins et al. (2015). For the case of Collins et al. (2015), there was a mature swell (wave age > 1) impending on thick, possibly compressed, pack ice. For our present case, the incident wave field was a highly forced (winds exceeding $15 \mathrm{~m} / \mathrm{s}$ ) and young wind sea, with a wave age (ratio of wave phase speed to wind speed) close to 1 . The sea ice was thin, newly formed frazil and pancake. Collins et al. (2015) observed total blocking of wave energy before swell broke up the ice and then propagated relatively unimpeded. In the present study, the young waves and thin ice conditions lead to attenuation of the high frequency waves (Rogers et al., 2016), with little effect on the peak of the wave spectrum. The different ice and wave conditions between these two studies prevents a direct comparison of the dynamics; more datasets with raw wave time series are required for a comprehensive analysis of linear versus nonlinear mechanisms for the formation of wave groups in sea ice.

\section{Conclusions}

Observations of wave groups in thin sea ice are explained by the attenuation of high frequency waves and the associated narrowing of the spectral bandwidth, relative to open water. Wave groups emerged as phase coherent interference out of an initially random wave field. The effect was reproduced in synthetic data from a linear model using the same bandwidth. The frequency dependence of wave attenuation in ice is thus a key parameter in this process because this effectively determines the spectral bandwidth within the ice. Underlying this result is the determination, specific to this dataset, that the deep water wave dispersion relation remains unchanged from open water to ice cover (Collins et al., 2018). Other combinations of wave and ice conditions might have strong changes in the dispersion relation, and these could result in focusing of wave energy leading to non-Guassian statistics and significant nonlinearity (Janssen \& Herbers, 2009). In particular, thicker, older, more compressed ice likely changes wave dynamics more significantly and thus may induce nonlinear behavior. Here, linear mechanisms sufficiently explain wave groups in ice.

Acknowledgments Funding was provided by the Office of Naval Research as part of the Arctic Sea State DRI under awards N00014-13-1-0284 and N00014-17-1-2544. The AWAC measurements are made from a mooring maintained as part of the Beaufort Gyre Observing System, with annual servicing lead by Rick Krishfield at Woods Hole Oceanographic Instituion. Joe Talbert and Alex de Klerk build and maintain the SWIFT buoys at the University of Washington Applied Physics Laboratory. Madison Smith shared code for making ice maps (Figure 1). Data are available at www.apl.uw.edu/ arcticseastate.

\section{References}

Banner, M. L., Barthelemy, X., Fedele, F., Allis, M., Benetazzo, A., Dias, F., \& Peirson, W. L. (2014). Linking reduced breaking crest speeds to unsteady nonlinear water wave group behavior. Physical Review Letters, 112, 114502. https://doi.org/10.1103/PhysRevLett.112.114502 Banner, M. L., \& Pierson, W. L. (2007). Wave breaking onset and strength for two-dimensional deep water wave groups. Journal of Fluid Mechanics, 585, 93-115.

Benjamin, T. B., \& Feir, J. (1967). The disintegration of wave trains on deep water part 1. Journal of Fluid Mechanics, 27(03), 417-430.

Cheng, S., Rogers, W. E., Thomson, J., Smith, M., Doble, M. J., Wadhams, P., et al. (2017). Calibrating a viscoelastic sea ice model for wave propagation in the arctic fall marginal ice zone. Journal of Geophysical Research: Oceans, 122, 8770-8793. https://doi.org/10.1002/ 2017JC013275

Collins, C. O., Doble, M., Lund, B., \& Smith, M. (2018). Observations of surface wave dispersion in the marginal ice zone. Journal of Geophysical Research: Oceans, 123, 3336-3354. https://doi.org/10.1029/2018JC013788

Collins, C. O., Rogers, W. E., Marchenko, A., \& Babanin, A. V. (2015). In situ measurements of an energetic wave event in the arctic marginal ice zone. Geophysical Research Letters, 42, 1863-1870. https://doi.org/10.1002/2015GL063063

Elgar, S., Guza, R. T., \& Seymour, R. J. (1984). Groups of waves in shallow water. Journal of Geophysical Research, 89(C3), 3623-3634.

Elgar, S., Guza, R. T., \& Seymour, R. J. (1985). Wave group statistics from numerical simulations of a random sea. Applied Ocean Research, $7(2), 93-96$.

Funke, E. R., \& Mansard, E. P. D. (1980). On the synthesis of realistic sea states. In Proceedings of the international conference on coastal engineering, pp. 2974-2991.

Gemmrich, J., \& Thomson, J. (2017). Observations of the shape and group dynamics of rogue waves. Geophysical Research Letters, 44, 1823-1830. https://doi.org/10.1002/2016GL072398

Herbers, T. H. C., Jessen, P. F., Janssen, T. T., Colbert, D. B., \& MacMahan, J. H. (2012). Observing ocean surface waves with GPS- tracked buoys. Journal of Atmospheric and Oceanic Technology, 29, 944-959. https://doi.org/10.1175/JTECH-D-11-00128.1

Janssen, P. (2003). Nonlinear four-wave interactions and freak waves. Journal of Physical Oceanography, 33(4), 863-884. https://doi.org/ 10.1175/1520-0485(2003)33<863:NFIAFW >2.0.CO;2 
Janssen, T., \& Herbers, T. H. C. (2009). Nonlinear wave statistics in a focal zone. Journal of Physical Oceanography, 39(8), 1948-1964. https://doi.org/10.1175/2009JPO4124.1

Kuik, A. J., Vledder, G. P. V., \& Holthuijsen, L. H. (1988). A method for the routine analyis of pitch-and-roll buoy wave data. Journal of Physical Oceanography, 18, 1020-1035.

Liu, A. K., \& Mollo-Christensen, E. (1988). Wave propagation in a solid ice pack. Journal of Physical Oceanography, 18(11), 1702-1712.

Longuet-Higgins, M. (1984). Statistical properties of wave groups in a random sea state. Philosophical Transactions of the Royal Society of London A: Mathematical, Physical and Engineering Sciences, 312(1521), 219-250. https://doi.org/10.1098/rsta.1984.0061

Magnell, B., Ivanov, L., \& Siegel, E. (2010). Measurements of ice parameters in the Beaufort sea using the Nortek AWAC acoustic Doppler current profiler. In Proceedings of the Oceans 2010 conference, MTS/IEEE, Seattle, WA (USA).

Pederson, T., Siegel, E., \& Wood, J. (2007). Directional wave measurements from a subsurface buoy with an acoustic wave and current profiler (awac). In Proceedings oceans 2007, Vancouver, Canada.

Persson, O., Blomquist, B., Guest, P., Stammerjohn, S., Fairall, C., Rainville, L., et al. (2018). Shipboard observations of the meteorology and near-surface environment during autumn freezeup in the beaufort/chukchi seas. Journal of Geophysical Research: Oceans, 123, 4930-4969. https://doi.org/10.1029/2018JC013786

Phillips, O. M. (1985). Spectral and statistical properties of the equilibrium range in wind-generated gravity waves. Journal of Fluid Mechanics, 156, 495-531.

Polnikov, V. G., \& Lavrenov, I. V. (2007). Calculation of the nonlinear energy transfer through the wave spectrum at the sea surface covered with broken ice. Oceanology, 47(3), 334-343. https://doi.org/10.1134/S0001437007030058

Rogers, W. E., Posey, P., Li, L., \& Allard, R. A. (2018). Forecasting and hindcasting waves in and near the marginal ice zone: Wave modeling and the onr "sea state" field experiment (NRL/MR/7320-18-9786): Naval Research Laboratory. https://www7320.nrlssc.navy.mil/pubs. php

Rogers, W. E., Thomson, J., Shen, H. H., Doble, M. J., Wadhams, P., \& Cheng, S. (2016). Dissipation of wind waves by pancake and frazil ice in the autumn beaufort sea. Journal of Geophysical Research: Oceans, 121, 7991-8007. https://doi.org/10.1002/2016JC012251

Serio, M., Onorato, M., R a Osborne, A., \& Janssen, P. (2005). On the computation of the benjamin-feir index. Nuovo Cimento della Societa Italiana di Fisica C, 28, 893-903. https://doi.org/10.1393/ncc/i2005-10134-1

Smith, M., Stammerjohn, S., Persson, O., Rainville, L., Liu, G., Perrie, W., et al. (2018). Episodic reversal of autumn ice advance caused by release of ocean heat in the beaufort sea. Journal of Geophysical Research: Oceans, 123, 3164-3185. https://doi.org/10.1002/2018JC013764

Smith, M., \& Thomson, J. (2019). Ocean surface turbulence in newly formed marginal ice zones. Journal of Geophysical Research: Oceans, 124, 1382-1398. https://doi.org/10.1029/2018JC014405

Stopa, J. E., Ardhuin, F., Thomson, J., Smith, M. M., Kohout, A., Doble, M., \& Wadhams, P. (2018). Wave attenuation through an arctic marginal ice zone on 12 october 2015: 1. measurement of wave spectra and ice features from sentinel 1a. Journal of Geophysical Research: Oceans, 123, 3619-3634. https://doi.org/10.1029/2018JC013791

Sullivan, P. P., Banner, M. L., Morison, R. P., \& Peirson, W. L. (2018). Turbulent flow over steep steady and unsteady waves under strong wind forcing. Journal of Physical Oceanography, 48(1), 3-27. https://doi.org/10.1175/JPO-D-17-0118.1

Thomson, J. (2012). Wave breaking dissipation observed with SWIFT drifters. Journal of Atmospheric and Oceanic Technology, 29(12), 1866-1882. https://doi.org/10.1175/JTECH-D-12-00018.1

Thomson, J., Ackley, S., Girard-Ardhuin, F., Ardhuin, F., Babanin, A., Boutin, G., et al. (2018). Overview of the arctic sea state and boundary layer physics program. Journal of Geophysical Research: Oceans, 123, 8674-8687. https://doi.org/10.1002/2018JC013766

Thomson, J., Girton, J. B., Jha, R., \& Trapani, A. (2018). Measurements of directional wave spectra and wind stress from a wave glider autonomous surface vehicle. Journal of Atmospheric and Oceanic Technology, 35(2), 347-363. https://doi.org/10.1175/ JTECH-D-17-0091.1

Timco, G. W., \& Weeks, W. F. (2010). A review of the engineering properties of sea ice. Cold Regions Science and Technology, 60(2), 107-129. https://doi.org/10.1016/j.coldregions.2009.10.003

Toffoli, A., Gramstad, O., Trulsen, K., Monbaliu, J., Bitner-Gregersen, E., \& Onorato, M. (2010). Evolution of weakly nonlinear random directional waves: laboratory experiments and numerical simulations. Journal of Fluid Mechanics, 664, 313-336. https://doi.org/10.1017/ S002211201000385X

van den Bremer, T. S., \& Taylor, P. H. (2015). Estimates of lagrangian transport by surface gravity wave groups: The effects of finite depth and directionality. Journal of Geophysical Research: Oceans, 120, 2701-2722. https://doi.org/10.1002/2015JC010712

WAFO-group (2000). WAFO - a matlab toolbox for analysis of random waves and loads - a tutorial [Computer software manual]. Lund, Sweden. http://www.maths.lth.se/matstat/

Waseda, T., Kinoshita, T., \& Tamura, H. (2009). Evolution of a random directional wave and freak wave occurrence. Journal of Physical Oceanography, 39(3), 621-639. https://doi.org/10.1175/2008JPO4031.1 